Received: 27 March 2017

Accepted: 12 June 2017

Published online: 26 October 2017

\section{Recommendation for incorporation of a different lymph node scoring system in future AJCC $\mathrm{N}$ category for oral cancer}

\author{
Ching-Chih Lee ${ }^{1,2,3,4,5}$, Yu-Chieh Su ${ }^{6,7}$, Shih-Kai Hung ${ }^{8,9}$, Po-Chun Chen ${ }^{10}$, Chung-I. Huang ${ }^{11}$, \\ Wei-Lun Huang ${ }^{12}$, Yu-Wei Lin ${ }^{13,14}$ \& Ching-Chieh Yang $\mathbb{1}^{13,14,15}$
}

To compare the prognostic value of 3 different lymph node scoring systems " log odds of positive nodes (LODDS), lymph node ratio ( $\mathrm{rN}$ ), and lymph node yield "in an effort to improve the staging of oral cancer. We identified 3958 oral cancer patients from Surveillance, Epidemiology, and End Results database from 2007 to 2013. In univariate analysis, LODDS, pN, rN, and lymph node yield were prognostic factors for 5 -year disease-specific survival (DSS) and overall survival (OS). Multivariate analysis indicated that patients with LODDS 4 had worst 5-year DSS and OS. Stage migration occurred in $\mathrm{pN} 1$ and $\mathrm{pN} 2$ patients with LODDS 4 . In pN1 patients, those with LODDS 4 had the worst 5-year DSS (41.2\%) and OS (31.6\%) than patients with pN1 and LODDS 2-3. In pN2 patients, those with LODDS4 had the worst 5-year DSS (34.5\%) and OS (27.4\%) than patients with pN2 and LODDS 2-3. The proposed staging system, which incorporates LODDS with AJCC $\mathrm{pN}$, had better discriminability and prediction accuracy for predicting survival. We also noted that patients with LODDS 4 given adjuvant radiotherapy had better 5-year DSS and OS. The LODDS should be considered as a future candidate measurement for $\mathrm{N}$ category in oral cancer.

Most oncologists stage oral cancer using the American Joint Committee on Cancer (AJCC) tumor, node, metastasis (TNM) system, and also use this system for clinical decision-making and development of therapeutic strategies $^{1}$. Although the most recent (8th edition) TNM classification for oral cancer, which considers extra-capsular extension, will be introduced in the near future, the impact of other clinical and pathological factors on survival indicates the need for a better staging tool ${ }^{2,3}$. One of the most important factors are lymph node number status, such as total lymph nodes retrieved, ratio of positive lymph nodes ( $\mathrm{rN})$, and log odds of positive lymph nodes $(\text { LODDS })^{4-6}$. Therefore, a study of the prognostic value of different lymph node scoring systems in oral cancer may aide in development of the forthcoming staging system.

Many studies have confirmed that the number of evaluated lymph nodes correlates with outcomes, and recommend that at least 18 lymph nodes be examined in patients with oral cancer ${ }^{6,7}$. Patients with inadequate lymph node harvests might experience stage migration and subsequent underestimation of disease severity ${ }^{8,9}$. Besides lymph node yield, other studies have analyzed the prognostic impact of $\mathrm{rN}$-the ratio of positive lymph nodes

\footnotetext{
${ }^{1}$ Department of Otolaryngology, Head and Neck Surgery, Kaohsiung Veterans General Hospital, Kaohsiung, Taiwan. ${ }^{2}$ School of Medicine, National Defense Medical Center, Taipei, Taiwan. ${ }^{3}$ Department of Otolaryngology, Head and Neck Surgery, Tri-Service General Hospital, Taipei, Taiwan. ${ }^{4}$ Institute of Hospital and Health Care Administration, National Yang-Ming University, Taipei, Taiwan. ${ }^{5}$ School of Medicine, National Yang-Ming University, Taipei, Taiwan. ${ }^{6}$ Department of Hematology and Oncology, E-Da hospital, Kaohsiung, Taiwan. ${ }^{7}$ School of Medicine, College of Medicine, I-Shou University, Kaohsiung, Taiwan. ${ }^{8}$ Department of Radiation Oncology, Buddhist Dalin Tzu Chi Hospital, Chiayi, Taiwan. ${ }^{9}$ School of Medicine, Tzu Chi University, Hualian, Taiwan. ${ }^{10}$ Department of Radiation Oncology, Pingtung Christian Hospital, Pingtung, Taiwan. ${ }^{11}$ Department of Radiation Oncology, E-Da Cancer Hospital, Kaohsiung, Taiwan. ${ }^{12}$ Department of Radiation oncology, Kaohsiung Veterans General Hospital, Kaohsiung, Taiwan. ${ }^{13}$ Department of Radiation Oncology, Chi-Mei Medical Center, Tainan, Taiwan. ${ }^{14}$ Institute of Biomedical Sciences, National Sun Yat-Sen University, Kaohsiung, Taiwan. ${ }^{15}$ Department of Pharmacy, Chia-Nan University of Pharmacy and Science, Tainan, Taiwan. Correspondence and requests for materials should be addressed to C.-C.Y. (email: cleanclear0905@gmail.com)
} 
to total examined nodes-in the staging of oral cancer $^{4,10}$. In fact, the $\mathrm{rN}$ category is a reliable predictor of oral cancer outcomes ${ }^{4}$. Recent studies indicate that the LODDS may provide a more accurate prediction of survival than the AJCC $\mathrm{pN}$ and $\mathrm{rN}$ categories ${ }^{11-13}$. In particular, LODDS can discriminate among patients who have the same ratio of node metastasis but different survival rates, especially patients without positive lymph nodes or with an insufficient number of retrieved nodes ${ }^{11,14}$. Although there is limited literature on the use of LODDS in oral cancer, our prior studies showed that LODDS had better discriminability of oral cancer patients from different single institution ${ }^{5,15}$.

The objective of the current study of oral cancer patients was to use multivariate analysis to compare different measures of lymph node status-LODDS, AJCC pN, rN, and lymph node yield-to identify the system with the greatest prognostic power. We used the Surveillance, Epidemiology, and End Results (SEER) database to compare all features of patients, so that the large number of cases provides sufficient statistical power. We also propose a new staging system that can identify the high-risk group using an independent factor to adjust risk features for a future prospective study.

\section{Results}

Demographic data. Table 1 summarizes the demographic and clinical characteristics of the study cohort. There were 3958 cases with newly diagnosed oral cancer (2528 men [63.9\%] and 1430 women [36.1\%]). The mean age at diagnosis was $59 \pm 13$ years. About $52 \%$ of the patients had tongue cancer and $25 \%$ had inadequate lymph node dissection. The mean lymph node yield was $33 \pm 17$, and the mean number of positive lymph nodes was $1.31 \pm 2.65$

Univariate and multivariate analysis of different lymph node measurements. Before our analysis, the multicollinearity and the reciprocal action effects among LODDS, AJCC pN, rN, and lymph node yield have been checked and presented no interaction between these measurements (Supplementary Table 1). Univariate analysis indicated that LODDS, $\mathrm{pN}, \mathrm{rN}$, and regional lymph nodes were significantly associated with 5-year DSS and OS (Fig. 1 and Supplementary Table 2). However, multivariate analysis indicated that only LODDS and $\mathrm{rN}$ measurements were significantly associated with 5-year OS (Table 2). In particular, patients with LODDS4 had poorer 5-year DSS (HR: 1.91, 95\% CI: 1.28-2.83) and OS (HR: 1.86, 95\% CI: 1.32-2.61) than those with LODDS1-3. Patients classified as rN3 had the worst 5-year OS (HR: 4.10, 95\% CI, 0.93-18.19).

Stage migration in different $\mathrm{pN}$ categories. We performed stratified analysis to check whether stage migration developed in the different $\mathrm{pN}$ and LODDS groups (Table 3 and Fig. 2). In pN0 patients, the LODDS1 and LODDS2 groups had similar survival rates; in pN1-2 patients, the LODDS4 group had the worst survival rate. Stage migration occurred in $\mathrm{pN} 1$ and $\mathrm{pN} 2$ patients with LODDS 4 . In pN1 patients, those with LODDS4 had the worst 5-year DSS (41.2\%) and OS (31.6\%) than patients with pN1 and LODDS2-3. In pN2 patients, those with LODDS4 had the worst 5-year DSS (34.5\%) and OS (27.4\%) than patients with pN2 and LODDS2-3. We also examined the presence of stage migration for $\mathrm{rN}$ according to $\mathrm{pN}$ status, but observed no stage migration, presumably because only a few patients had $\mathrm{rN} 3$ disease (Supplementary Table 3 ).

Performance of AJCC TNM stage and hypothetical system. Due to the effect of stage migration of $\mathrm{pN} 1$ and $\mathrm{pN} 2$ cancer, modification of $\mathrm{N}$ category was proposed, similar to our previous literature (Table 3$)^{15}$. As described in the 'Material and Methods', subgroups with fewer than 30 OSCC patients were not included in this analysis. This led to a new $\mathrm{N}$ category in which each patient was placed into one of four groups: new N0 (pN0 and LODDS1-2); new N1 (pN1 and LODDS2-3); new N2 (pN1 and LODDS4, pN2 and LODDS2-3); and new N3 (pN2 and LODDS4). Thus, we compared the stage-specific DSS and OS survival rates according to current AJCC TNM staging and our new system (Fig. 3). There was no difference in DSS $(p=0.63)$ and OS $(p=0.985)$ between the AJCC stage IVa and IVb disease. However, the new system discriminated stage IVa and IVb in terms of DSS $(p<0.001)$ and OS $(p<0.001)$. In prediction of DSS, the new system outperformed the AJCC TNM system with a higher linear chi-square value (397 vs. 327), a lower AIC (13275 vs. 13329), and a higher Harrell's C statistic (0.722 vs. 0.703) (Table 4). Similarly, a comparison of these 2 systems in terms of OS indicated the new system had higher linear chi-square (407 vs. 326), a lower AIC (17454 vs. 17514), and a higher Harrell's C statistic (0.692 vs. $0.673)$. These results were in agreement with our previous single center study ${ }^{15}$. Moreover, our multivariate analysis indicated these results were robust (Table 5). Thus, the new model had better discriminatability and better prediction of DSS and OS than the existing AJCC TNM system. Supplementary Tables 4 and 5 show the detailed results of the Cox regression model.

Role of adjuvant radiotherapy for high-risk patients. We further analyzed the effect of adjuvant radiotherapy in patients with different LODDS. Among patients with LODDS 4, those treated with adjuvant radiotherapy had better 5-year DSS (aHR: 0.56, 95\% CI: 0.42-0.73) and OS (aHR: 0.52; 95\% CI: 0.41-0.67) than those not receiving adjuvant radiotherapy after adjusting other factors (Fig. 4).

\section{Discussion}

We comprehensively analyzed the use of different lymph node scoring systems in patients with oral cancer using the SEER database, and validated the prognostic independency of LODDS for oral cancer in the United States. In particular, oral cancer patients with LODDS 4 (LODDS $>-0.88$ ) had 91\% greater mortality in our 5-year DSS analysis and $86 \%$ greater mortality in our 5 -year OS analysis relative to those with LODDS1 (LODDS $\leq-1.68$ ) after adjustment for confounding. Stage migration occurred in pN1 and in pN2 with LODDS 4. The new system had better discriminability and prediction accuracy than the existing AJCC TNM system. Furthermore, our multivariate analysis indicated that high-risk patients (LODDS 4) benefitted from adjuvant radiotherapy. LODDS is 


\begin{tabular}{|c|c|}
\hline Variables & Number, $\boldsymbol{n}(\%)$ \\
\hline Age $($ Mean $\pm \mathrm{SD})$ & $59 \pm 13$ \\
\hline \multicolumn{2}{|l|}{ Gender } \\
\hline Male & $2528(63.9 \%)$ \\
\hline Female & $1430(36.1 \%)$ \\
\hline \multicolumn{2}{|l|}{ Race } \\
\hline White & $3316(83.8 \%)$ \\
\hline Black/other & $642(16.2 \%)$ \\
\hline \multicolumn{2}{|l|}{ Marital status } \\
\hline Married & $2240(56.6 \%)$ \\
\hline Other status & $1718(43.4 \%)$ \\
\hline \multicolumn{2}{|l|}{ Tumor subsite } \\
\hline Tongue & $2041(51.6 \%)$ \\
\hline Lip & $160(4.0 \%)$ \\
\hline Floor of mouth & $671(17.0 \%)$ \\
\hline Gum and retromolar trigone & $680(17.2 \%)$ \\
\hline Buccal mucosa & $268(6.8 \%)$ \\
\hline Hard palate & $55(1.4 \%)$ \\
\hline Other areas & $83(2.1 \%)$ \\
\hline \multicolumn{2}{|l|}{ Differentiation } \\
\hline Well/moderately & $3060(77.3 \%)$ \\
\hline Poorly/undifferentiated & $898(22.7 \%)$ \\
\hline \multicolumn{2}{|l|}{ Regional lymph nodes examined } \\
\hline Adequate & $2969(75.0 \%)$ \\
\hline Inadequate & $989(25.0 \%)$ \\
\hline \multicolumn{2}{|l|}{ AJCC pT } \\
\hline $\mathrm{T} 1$ & $1398(35.3 \%)$ \\
\hline $\mathrm{T} 2$ & $1353(34.2 \%)$ \\
\hline $\mathrm{T} 3$ & $474(12.0 \%)$ \\
\hline $\mathrm{T} 4$ & $733(18.5 \%)$ \\
\hline \multicolumn{2}{|l|}{ AJCC pN } \\
\hline No & $2132(53.9 \%)$ \\
\hline $\mathrm{N} 1$ & $826(20.9 \%)$ \\
\hline $\mathrm{N} 2$ & $967(24.4 \%)$ \\
\hline N3 & $33(0.8 \%)$ \\
\hline \multicolumn{2}{|l|}{ LODDS } \\
\hline LODDS1 (LODDS $\leqq-1.68)$ & $1360(34.4 \%)$ \\
\hline LODDS2 $(-1.68<$ LODDS $\leqq-1.29)$ & $1214(30.7 \%)$ \\
\hline LODDS3 $(-1.29<$ LODDS $\leqq-0.88)$ & $795(20.1 \%)$ \\
\hline LODDS4 ( $-0.88<$ LODDS) & $589(14.9 \%)$ \\
\hline \multicolumn{2}{|l|}{$\mathrm{rN}$} \\
\hline $\mathrm{N} 0(\mathrm{rN}=0)$ & $2139(54.0 \%)$ \\
\hline $\mathrm{N} 1(0<\mathrm{rN} \leqq 0.2)$ & $1626(41.1 \%)$ \\
\hline $\mathrm{N} 2(0.2<\mathrm{rN} \leqq 0.4)$ & $159(4.0 \%)$ \\
\hline $\mathrm{N} 3(0.4<\mathrm{rN})$ & $34(0.9 \%)$ \\
\hline \multicolumn{2}{|l|}{ Radiotherapy } \\
\hline No & $1851(46.8 \%)$ \\
\hline Yes & $2107(53.2 \%)$ \\
\hline \multicolumn{2}{|l|}{ Year of diagnosis } \\
\hline 2007 & $432(10.9 \%)$ \\
\hline 2008 & $488(12.3 \%)$ \\
\hline 2009 & $588(14.9 \%)$ \\
\hline 2010 & $575(14.5 \%)$ \\
\hline 2011 & $587(14.8 \%)$ \\
\hline 2012 & $648(16.4 \%)$ \\
\hline 2013 & $640(16.2 \%)$ \\
\hline
\end{tabular}

Table 1. Demographic and clinical characteristics of the oral cancer patients, $n=3958$. Abbreviation: LODDS, log odds of positive lymph nodes; rN, ratio-based lymph node system. 
LODDS

DSS

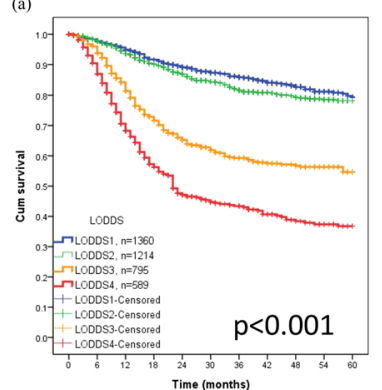

OS

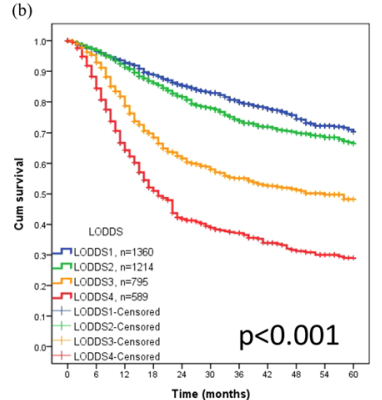

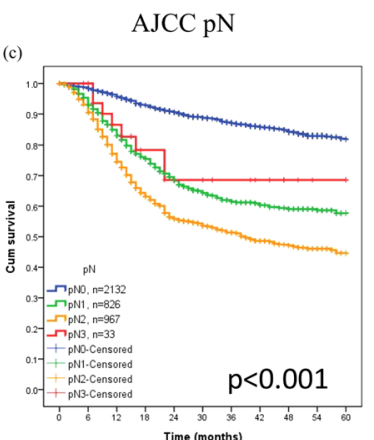

(d)

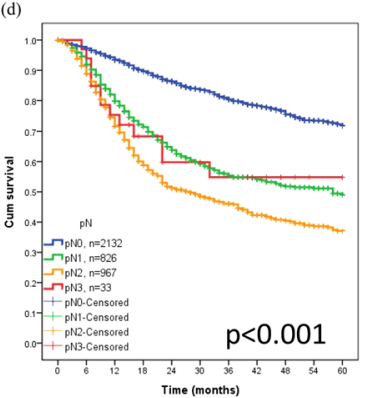

$\mathrm{rN}$

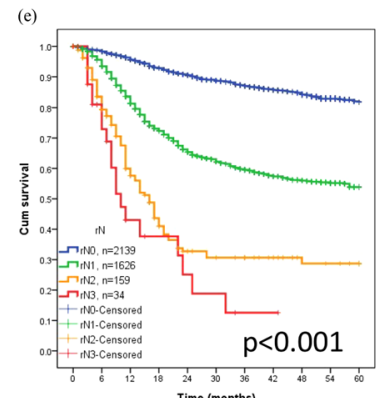

(f)

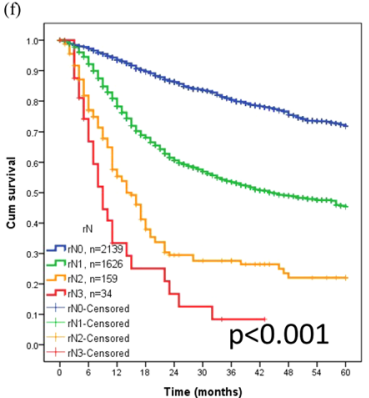

Regional lymph nodes examined (g)

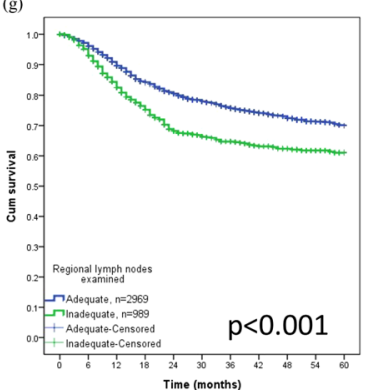

(h)

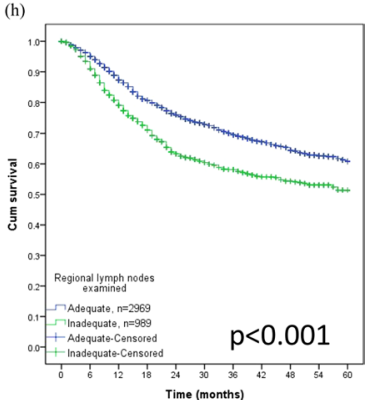

Figure 1. Kaplan-Meier curves for (a,b) LODDS in DSS and OS, (c,d) AJCC pN in DSS and OS, (e,f) rN in 5 -year DSS and OS, and regional lymph nodes examined (g,h) from Surveillance, Epidemiology, and End Results (SEER) database.

therefore a reliable method that can be easily used by clinical practitioners, and should be considered as a future candidate measurement for nodal classification of oral cancer.

Currently, oral cancer patients with positive lymph nodes are staged as AJCC stage III-IV, and recommended for adjuvant radiotherapy or chemo-radiotherapy ${ }^{16}$. Due to multiple shortcomings of the $\mathrm{N}$ category in the current AJCC TNM staging system ( 7 th edition), the new updated version ( $8^{\text {th }}$ edition) has incorporated extra-capsular spread in the clinical and pathological $\mathrm{N}$ category. However, there is continued discussion regarding the use of other prognostic factors, such as lymph node count, $\mathrm{rN}$, and LODDS, for reducing stage migration ${ }^{4,5,8}$. This motivated our present research to assess the use of different lymph node scoring systems to develop a new $\mathrm{N}$ staging category that better stratifies high-risk patients for more intense therapy.

Multiple studies have investigated lymph node count as a prognostic factor in stomach cancer, colon cancer, and head and neck cancer, and also as a potential quality metric for neck dissection ${ }^{7,8}$. Divi et al. performed a large cohort study to examine these associations using a nation-wide database from the United States ${ }^{8}$. Their results showed an independent and significant association between fewer than 18 lymph nodes examined and increased risk of death (HR: $1.18,95 \%$ CI: 1.13 to 1.22 ). In addition, when stratified by clinical nodal stage, there was an increased risk of death in the node-negative group (HR: 1.24, 95\% CI: 1.17 to 1.32) and the node-positive group (HR: 1.12; 95\% CI: 1.05 to 1.19 ). Thus, there is a significant overall survival advantage when more than 18 lymph nodes are examined after neck dissection. However, lymph node yield may depend on the specific hospitals, cancer severity, patient age, and patient performance status. Furthermore, the lymph node count may not reflect the real impact of positive lymph nodes on survival, so the lymph node ratio system should be still considered for $\mathrm{pN}$ stage assessment. The results of our univariate analysis showed a difference in DSS and OS according to LODDS, $\mathrm{pN}, \mathrm{rN}$, and regional lymph nodes examined (Fig. 1 and Supplementary Table 2). However, only LODDS and $\mathrm{rN}$ remained statistically significant in the multivariate analysis (Table 2). Thus, $\mathrm{rN}$ or LODDS system should be considered for improvement of the AJCC $\mathrm{pN}$ stage.

The current AJCC pN category for oral cancer is based on the size, number, and location of resected lymph nodes. However, when there is stage migration, the $\mathrm{pN}$ category underestimates the true extent of lymph node disease, and is therefore considered imperfect for prognostic purposes. For example, patients with the same $\mathrm{pN}$ classification, but a different number of examined nodes, will be given different prognoses. Therefore, the $\mathrm{rN}$ and LODDS systems, two new classifications for nodal estimation and behavior, are better than the traditional number-based $\mathrm{pN}$ system ${ }^{9,17}$. In our previous studies of oral cancer $^{5,15}$, we found that LODDS performed better than the $\mathrm{pN}$ and $\mathrm{rN}$ systems. The main reason is that there is a non-linear association between the LODDS distribution and number of pathologically positive nodes ${ }^{11}$. Therefore, compared with $\mathrm{rN}$, LODDS can discriminate among patients without positive lymph nodes or with a few positive nodes when insufficient nodes are retrieved. For example, a pN1 patient with a high LODDS should not be treated the same as a pN1 patient with low LODDS, because the former has a higher risk of occult metastases and worse prognosis. In this context, it is noteworthy that LODDS was a reliable lymph node measurement, and may be considered an alternative to $\mathrm{pN}$ stage.

Previous studies have successfully used LODDS in the study of breast, gastric, and colorectal cancer ${ }^{11,17,18}$. Besides our own former experience from two specific hospitals, there is little data on use of LODDS on outcome from oral cancer. Yildiz et al. reported a study of 225 surgically treated head and neck cancer patients, and 


\begin{tabular}{|c|c|c|c|c|c|c|}
\hline \multirow[b]{2}{*}{ Variables } & \multicolumn{3}{|c|}{ Disease-specific survival } & \multicolumn{3}{|c|}{ Overall survival } \\
\hline & HR & 95\% CI & p value & HR & $95 \% \mathrm{CI}$ & p value \\
\hline Age & 1.02 & $(1.01-1.02)$ & $<0.0001$ & 1.02 & $(1.02-1.03)$ & $<0.0001$ \\
\hline \multicolumn{7}{|l|}{ Race } \\
\hline White & 1 & & & & & \\
\hline Black/other & 1.11 & $(0.93-1.32)$ & 0.250 & & & \\
\hline \multicolumn{7}{|l|}{ Marital status } \\
\hline Married & 1 & & & 1 & & \\
\hline Other status & 1.11 & $(0.97-1.28)$ & 0.118 & 1.25 & $(1.11-1.41)$ & 0.000 \\
\hline \multicolumn{7}{|l|}{ Tumor subsite } \\
\hline Tongue & 1 & & & 1 & & \\
\hline Lip & 0.43 & $(0.27-0.70)$ & 0.001 & 0.57 & $(0.40-0.82)$ & 0.002 \\
\hline Floor of mouth & 1.08 & $(0.90-1.30)$ & 0.423 & 1.14 & $(0.97-1.33)$ & 0.123 \\
\hline $\begin{array}{l}\text { Gum and retromolar } \\
\text { trigone }\end{array}$ & 0.94 & $(0.78-1.14)$ & 0.551 & 0.98 & $(0.83-1.15)$ & 0.765 \\
\hline Buccal mucosa & 0.95 & $(0.73-1.24)$ & 0.700 & 0.87 & $(0.68-1.11)$ & 0.263 \\
\hline Hard palate & 1.09 & $(0.67-1.79)$ & 0.728 & 1.03 & $(0.65-1.62)$ & 0.913 \\
\hline Other areas & 1.16 & $(0.77-1.77)$ & 0.480 & 1.25 & $(0.88-1.78)$ & 0.215 \\
\hline \multicolumn{7}{|l|}{ Differentiation } \\
\hline Well/moderately & 1 & & & 1 & & \\
\hline Poorly/undifferentiated & 1.11 & $(0.96-1.29)$ & 0.171 & 1.15 & $(1.01-1.31)$ & 0.042 \\
\hline \multicolumn{7}{|c|}{ Regional lymph nodes examined } \\
\hline Adequate & 1 & & & 1 & & \\
\hline Inadequate & 0.89 & $(0.75-1.06)$ & 0.187 & 0.93 & $(0.80-1.08)$ & 0.317 \\
\hline \multicolumn{7}{|l|}{ AJCC pT } \\
\hline $\mathrm{T} 1$ & 1 & & & 1 & & \\
\hline $\mathrm{T} 2$ & 1.76 & $(1.45-2.14)$ & $<0.0001$ & 1.61 & $(1.37-1.90)$ & $<0.0001$ \\
\hline $\mathrm{T} 3$ & 3.04 & $(2.41-3.83)$ & $<0.0001$ & 2.79 & $(2.29-3.39)$ & $<0.0001$ \\
\hline $\mathrm{T} 4$ & 3.31 & $(2.67-4.10)$ & $<0.0001$ & 2.84 & $(2.36-3.42)$ & $<0.0001$ \\
\hline \multicolumn{7}{|l|}{ AJCC $\mathrm{pN}$} \\
\hline N0 & 1 & & & 1 & & \\
\hline $\mathrm{N} 1$ & 1.83 & $(0.45-7.39)$ & 0.397 & 1.20 & $(0.30-4.85)$ & 0.796 \\
\hline $\mathrm{N} 2$ & 1.85 & $(0.46-7.49)$ & 0.390 & 1.27 & $(0.31-5.11)$ & 0.740 \\
\hline N3 & 0.94 & $(0.20-4.50)$ & 0.941 & 0.87 & $(0.20-3.90)$ & 0.858 \\
\hline \multicolumn{7}{|l|}{ LODDS } \\
\hline LODDS1 & 1 & & & 1 & & \\
\hline LODDS2 & 0.86 & $(0.66-1.12)$ & 0.251 & 0.97 & $(0.79-1.20)$ & 0.800 \\
\hline LODDS3 & 1.36 & $(0.95-1.94)$ & 0.096 & 1.30 & $(0.96-1.77)$ & 0.089 \\
\hline LODDS4 & 1.91 & $(1.28-2.83)$ & 0.002 & 1.86 & $(1.32-2.61)$ & 0.000 \\
\hline \multicolumn{7}{|l|}{$\mathrm{rN}$} \\
\hline No & 1 & & & 1 & & \\
\hline N1 & 1.36 & $(0.33-5.59)$ & 0.668 & 1.72 & $(0.42-7.00)$ & 0.450 \\
\hline $\mathrm{N} 2$ & 2.48 & $(0.59-10.42)$ & 0.214 & 2.88 & $(0.69-11.97)$ & 0.145 \\
\hline N3 & 4.10 & $(0.93-18.19)$ & 0.063 & 5.25 & $(1.21-22.74)$ & 0.027 \\
\hline \multicolumn{7}{|l|}{ Radiotherapy } \\
\hline No & 1 & & & 1 & & \\
\hline Yes & \begin{tabular}{|l|l}
0.78 \\
\end{tabular} & $(0.67-0.91)$ & 0.002 & 0.71 & $(0.62-0.81)$ & $<0.0001$ \\
\hline
\end{tabular}

Table 2. Multivariate analysis for 5 -year disease-specific survival and overall survival, $n=3958 *$. Abbreviation: HR, hazard ratio; 95\% CI, 95\% confidence interval; LODDS, log odds of positive lymph nodes; rN, ratio-based lymph node system. *Variables with a $p$ value $<0.05$ in univairate analysis (Supplementary Table 2) were included in multivariate analysis.

reported LODDS as the only independent predictor for 5 -year OS when comparing $\mathrm{pN}$ and $\mathrm{rN}^{19}$. The present SEER study confirms and validates that LODDS especially LOODS 4 is an independent prognostic factor, among various lymph node assessments, for oral cancer in the United States. In pN1 patients, those with LODDS4 had the worst 5-year DSS (41.2\%) and OS (31.6\%) than patients with pN1 and LODDS2-3. In pN2 patients, those with LODDS4 had the worst 5-year DSS (34.5\%) and OS (27.4\%) than patients with pN2 and LODDS2-3. Therefore LODDS 4 can compensate for the effect of migration of the AJCC $\mathrm{pN}$ stage. Furthermore, previous studies have outlined the importance of lymph node yield ${ }^{7,8}$. What is the value of LODDS in modification of oral cancer 


\begin{tabular}{|c|c|c|c|c|c|c|c|c|c|}
\hline \multirow{2}{*}{$\begin{array}{l}\text { New N } \\
\text { category }\end{array}$} & \multirow[b]{2}{*}{ Inclusion } & \multirow[b]{2}{*}{ AJCC $\mathrm{pN}$} & \multirow[b]{2}{*}{ LODDS } & \multicolumn{3}{|c|}{ Disease-specific survival } & \multicolumn{3}{|c|}{ Overall survival } \\
\hline & & & & Case & Events & Survival rate (\%) & Case & Events & Survival rate (\%) \\
\hline \multirow{4}{*}{ No } & $\checkmark$ & pN0 & LODDS1 & 1331 & 159 & $80.3 \%$ & 1331 & 239 & $71.1 \%$ \\
\hline & $\checkmark$ & pN0 & LODDS2 & 801 & 78 & $84.3 \%$ & 801 & 137 & $73.1 \%$ \\
\hline & & pN0 & LODDS3 & 0 & 0 & - & 0 & 0 & - \\
\hline & & pN0 & LODDS4 & 0 & 0 & - & 0 & 0 & - \\
\hline \multirow{3}{*}{ N1 } & & pN1 & LODDS1 & 23 & 9 & $33.8 \%$ & 23 & 10 & $29.6 \%$ \\
\hline & $\checkmark$ & $\mathrm{pN} 1$ & \begin{tabular}{|l|} 
LODDS2 \\
\end{tabular} & 314 & 72 & $66.5 \%$ & 314 & 98 & $54.8 \%$ \\
\hline & $\checkmark$ & $\mathrm{pN1}$ & LODDS3 & 375 & 119 & $57.3 \%$ & 375 & 139 & $51.3 \%$ \\
\hline \multirow{4}{*}{ N2 } & $\checkmark$ & $\mathrm{pN1}$ & LODDS4 & 114 & 54 & $41.2 \%$ & 114 & 65 & $31.6 \%$ \\
\hline & & $\mathrm{pN} 2$ & LODDS1 & 6 & 2 & $60.0 \%$ & 6 & 2 & $60.0 \%$ \\
\hline & $\checkmark$ & $\mathrm{pN} 2$ & LODDS2 & 93 & 25 & $63.1 \%$ & 93 & 33 & $49.1 \%$ \\
\hline & $\checkmark$ & $\mathrm{pN} 2$ & \begin{tabular}{|l|} 
LODDS3 \\
\end{tabular} & 407 & 132 & $51.8 \%$ & 407 & 152 & $45.1 \%$ \\
\hline \multirow[t]{5}{*}{ N3 } & $\checkmark$ & $\mathrm{pN} 2$ & \begin{tabular}{|l} 
LODDS4 \\
\end{tabular} & 461 & 223 & $34.5 \%$ & 461 & 263 & $27.4 \%$ \\
\hline & & pN3 & \begin{tabular}{|l|} 
LODDS1 \\
\end{tabular} & 0 & 0 & - & 0 & 0 & - \\
\hline & & pN3 & \begin{tabular}{|l|} 
LODDS2 \\
\end{tabular} & 6 & 0 & - & 6 & 1 & $66.7 \%$ \\
\hline & & pN3 & \begin{tabular}{|l} 
LODDS3 \\
\end{tabular} & 13 & 5 & $54.0 \%$ & 13 & 7 & $44.9 \%$ \\
\hline & & pN3 & LODDS4 & 14 & 3 & $71.8 \%$ & 14 & 5 & $61.1 \%$ \\
\hline
\end{tabular}

Table 3. The 5-year overall survival and disease-specific survival of the oral cancer patients according to different AJCC $\mathrm{pN}$ plus LODDS, $n=3958$. Abbreviation: LODDS, log odds of positive lymph nodes.

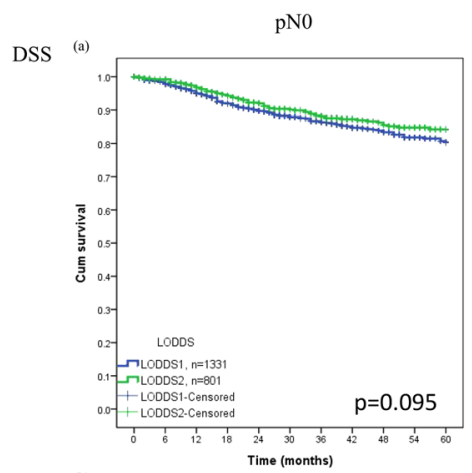

OS

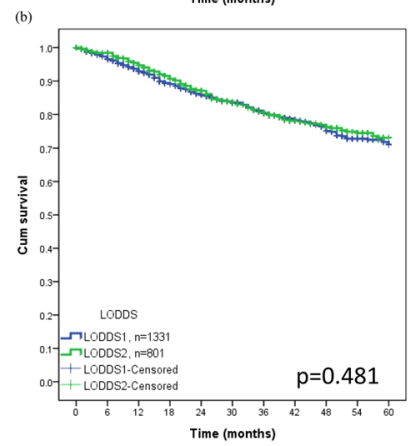

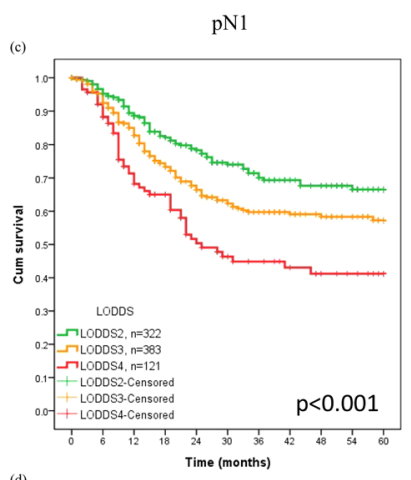

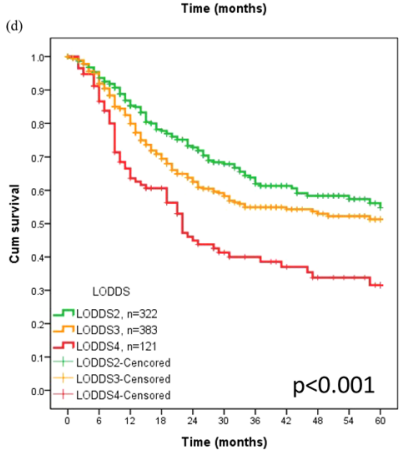

$\mathrm{pN} 2$
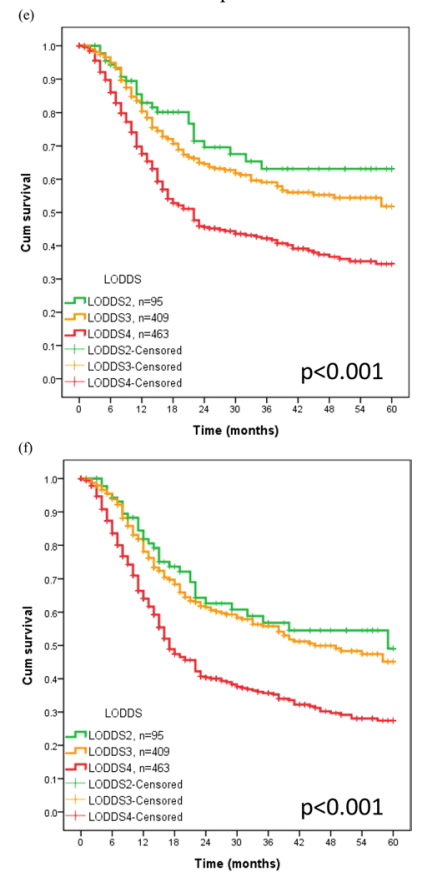

Figure 2. Kaplan-Meier curves for 5-year DSS and OS according to different AJCC pN plus LODDS.

staging system? Our multivariate analysis indicated the lymph node yield was not an independent prognosticator when LODDS was in the model simultaneously. Furthermore, LODDS has better discriminability then lymph node yield and patients with inadequate node dissection had worse survival ${ }^{8}$. The current literature provides little advice for oral cancer patients who received inadequate node dissection. In the present study, LODDS helped to stratify patients and select the most appropriate therapy for those with LODDS4 (LODDS $>-0.88$, who had better prognosis when adjuvant radiotherapy was administered. In summary, lymph node yield could be regarded as "quality measure" of neck dissection, just as "surgical margin" may be a proxy of surgical technique. However, LODDS can be regarded as an reliable lymph node measurement for oral cancer, and an indicator of the suitability for adjuvant therapy.

There are several limitations in this study. First, the SEER database provides no information on whether the patients underwent bilateral neck dissection. We tried to minimize this confounding effect in lymph node yield, through exclusion of patients with $\mathrm{pN} 2 \mathrm{c}$ disease, as previously suggested ${ }^{6,20}$. Moreover, we are currently conducting 
DSS

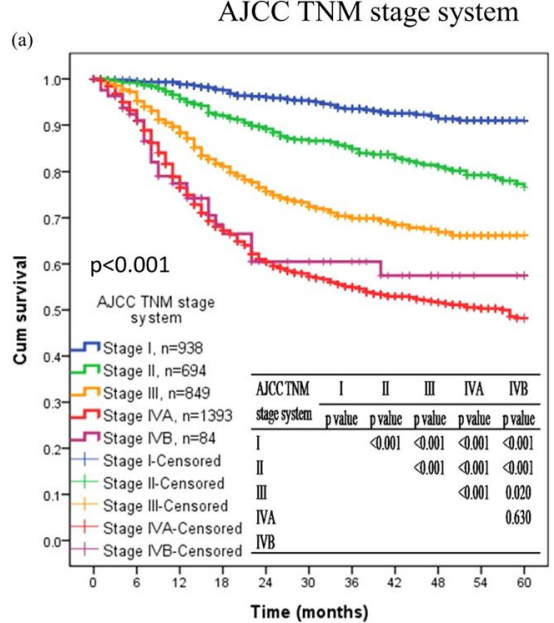

OS

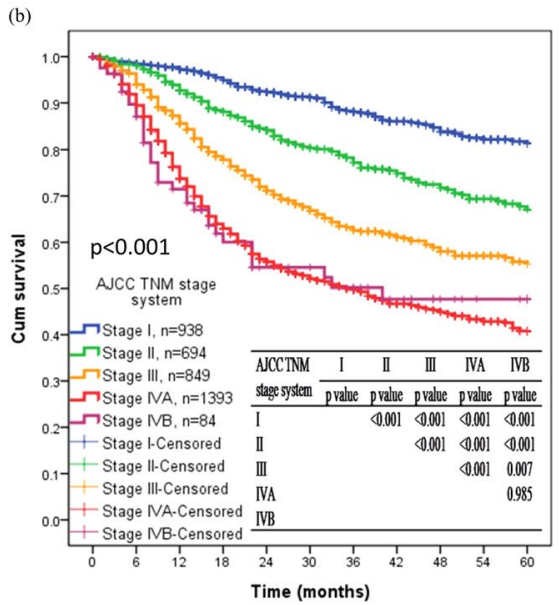

T-new N-M stage system
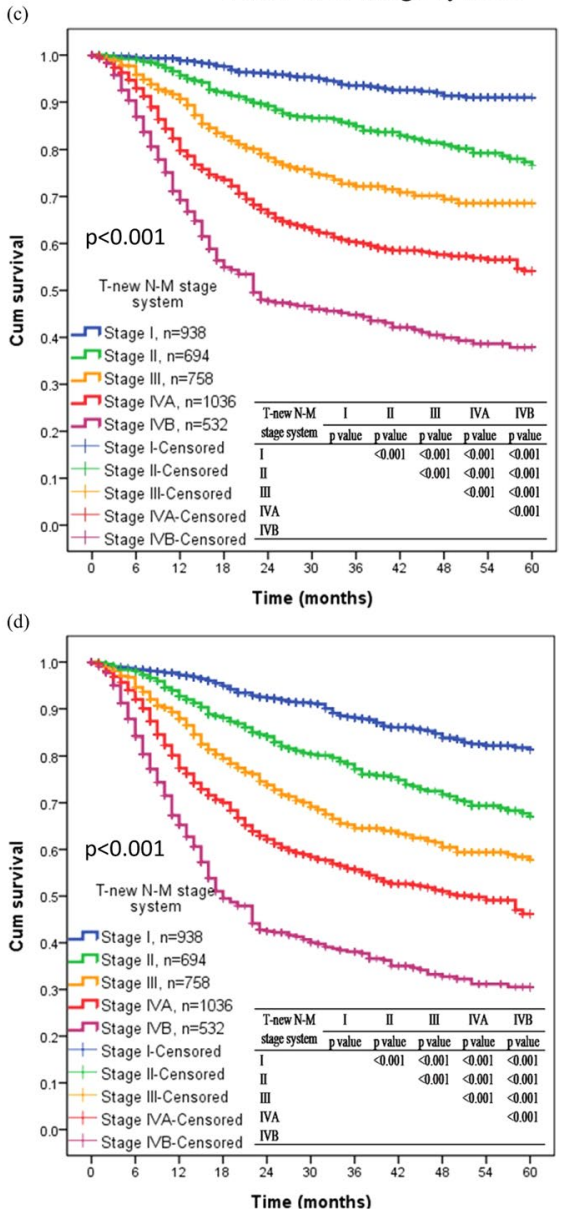

Figure 3. Kaplan-Meier curves for (a,b) TNM stage in DSS and OS, and (c,d) hypothetical T-new N-M system in 5-year DSS and OS.

\begin{tabular}{|l|l|l|l|l|l|}
\hline \multicolumn{2}{|l|}{ Subgroups } & Linear trend $\chi^{2}$ & AIC & Harrell's c-statistics \\
\hline Performance of DSS prediction & \multicolumn{1}{l|}{} \\
\hline AJCC TNM stage & I, II, III, IVA, IVB & 327 & 13329 & 0.703 \\
\hline T-New N-M stage & I, II, III, IVA, IVB & 397 & 13275 & 0.722 \\
\hline Performance of OS prediction & 326 & 17514 & 0.673 \\
\hline AJCC TNM stage & I, II, III, IVA, IVB & 326 & 17454 & 0.692 \\
\hline T-New N-M stage & I, II, III, IVA, IVB & 407 & & \\
\hline
\end{tabular}

Table 4. Discriminatory ability between AJCC TNM stage and T- New N-M stage system, $n=3958$. Abbreviation: DSS, disease-specific survival; OS, overall survival; AIC, Akaike information criterion.

research using the Taiwan Cancer Database, which has clinical and pathological TNM data, and this may help to resolve this limitation. Second, the cutoff points for $\mathrm{rN}$ and LODDS were selected by our recent studies ${ }^{5,21}$. Modification of these cutoff points may be necessary to prevent subgroups with too few patients for analysis. Third, multivariable analysis indicated that lymph node yield was not an independent factor when LODDS was in the model. We did not perform further stratified analysis due to lack of statistical independence. Our study was not aimed to challenge the prognostic value of lymph node yield, but to find additional prognostic useful indicators. In fact, LODDS may be considered almost as a proxy of lymph node yield. Current guidelines recommend extensive extirpation of lymph nodes, without adverse damage to vessels and nerves, when performing neck dissection. Fourth, the SEER database provided no information on use of adjuvant chemotherapy, extra-capsular invasion and margin status. Some other unmeasured biases may also exist. Further research linked to Medicare claims (provide longitudinal utilization information for the cancer cases in SEER) or the use of instrumental variable analysis may help us to resolve these important issues ${ }^{22}$. Although this study describes the protective effect of adjuvant radiotherapy in those with LODDS4 after adjusting for confounding, these findings should be verified by a prospective study. Finally, the number of patients in some of our subgroups (pN0 with LODDS3-4; pN1 with 


\begin{tabular}{|c|c|c|c|c|c|c|}
\hline \multirow[b]{2}{*}{ Variables } & \multicolumn{3}{|c|}{ Model 1: AJCC TNM-based model } & \multicolumn{3}{|c|}{ Model 2: T-new N-M-based model } \\
\hline & HR & $95 \% \mathrm{CI}$ & p value & HR & $95 \% \mathrm{CI}$ & p value \\
\hline \multicolumn{7}{|l|}{ DSS performance } \\
\hline \multicolumn{7}{|l|}{ AJCC pN } \\
\hline No & 1 & & & & & \\
\hline $\mathrm{N} 1$ & 3.11 & $2.59-3.74$ & $<0.001$ & & & \\
\hline $\mathrm{N} 2$ & 4.38 & $3.66-5.24$ & $<0.001$ & & & \\
\hline N3 & 2.22 & $1.08-4.54$ & 0.029 & & & \\
\hline \multicolumn{7}{|l|}{ New $\mathrm{N}$ category } \\
\hline N0 & & & & 1 & & \\
\hline $\mathrm{N} 1$ & & & & 2.86 & $2.35-3.47$ & $<0.001$ \\
\hline $\mathrm{N} 2$ & & & & 3.53 & $2.89-4.30$ & $<0.001$ \\
\hline N3 & & & & 5.79 & $4.75-7.06$ & $<0.001$ \\
\hline \multicolumn{7}{|l|}{ Discriminatory ability } \\
\hline Linear trend $\chi^{2}$ & \multicolumn{3}{|l|}{273} & \multicolumn{3}{|l|}{316} \\
\hline AIC & \multicolumn{3}{|c|}{13164} & \multicolumn{3}{|c|}{13131} \\
\hline Harrell's c-statistic & \multicolumn{3}{|l|}{0.757} & \multicolumn{3}{|c|}{0.762} \\
\hline \multicolumn{7}{|l|}{ OS performance } \\
\hline \multicolumn{7}{|l|}{ AJCC $\mathrm{pN}$} \\
\hline No & 1 & & & & & \\
\hline N1 & 2.55 & $2.18-2.98$ & $<0.001$ & & & \\
\hline $\mathrm{N} 2$ & 3.55 & $3.05-4.14$ & $<0.001$ & & & \\
\hline N3 & 2.49 & $1.42-4.38$ & 0.001 & & & \\
\hline \multicolumn{7}{|l|}{ New $\mathrm{N}$ category } \\
\hline N0 & & & & 1 & & \\
\hline $\mathrm{N} 1$ & & & & 2.36 & $2.00-2.79$ & $<0.001$ \\
\hline $\mathrm{N} 2$ & & & & 2.83 & $2.38-3.36$ & $<0.001$ \\
\hline N3 & & & & 4.77 & $4.02-5.66$ & $<0.001$ \\
\hline \multicolumn{7}{|l|}{ Discriminatory ability } \\
\hline Linear trend $\chi^{2}$ & \multicolumn{3}{|l|}{279} & \multicolumn{3}{|l|}{335} \\
\hline AIC & \multicolumn{3}{|c|}{17243} & \multicolumn{3}{|c|}{17200} \\
\hline Harrell's c-statistic & \multicolumn{3}{|l|}{0.735} & \multicolumn{3}{|l|}{0.741} \\
\hline
\end{tabular}

Table 5. Multivariate analysis of 5-year disease-specific survival \& overall survival and model discrimination, $n=3958 *$ *Adjusted for age, gender, tumor subsite, AJCC pT, differentiation, radiotherapy, marital status, race and year of diagnosis. Abbreviation: DSS, disease-specific survival; OS, overall survival; HR, hazard ratio; 95\% CI, 95\% confidence interval; AIC, Akaike information criterion.

DSS

(a)

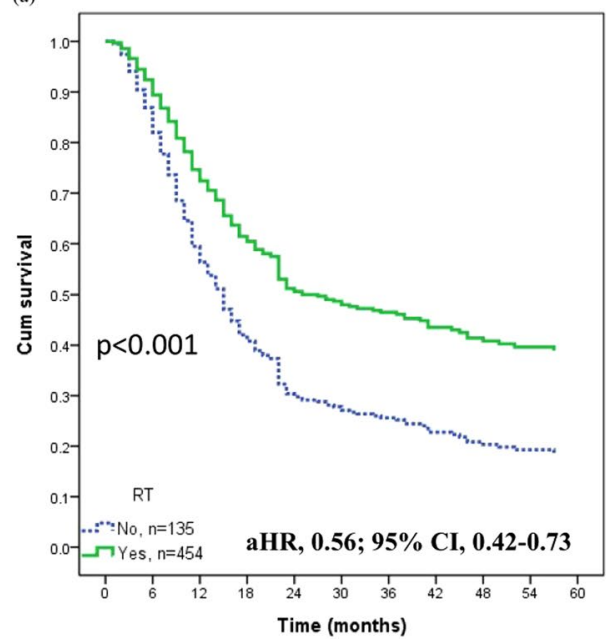

OS

(b)

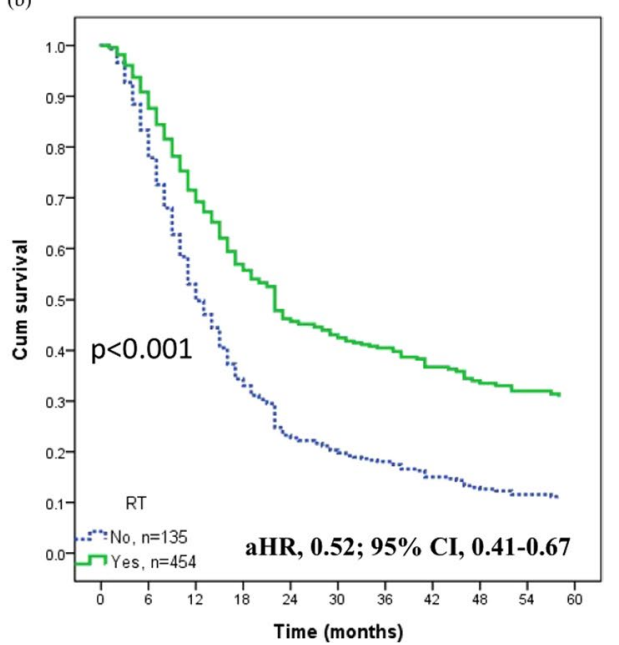

Figure 4. The adjusted Kaplan-Meier curves for adjuvant radiotherapy effect in 5-year DSS (a) and OS (b) among LODDS 4 patients. 
LODDS1; pN2 with LODDS1; pN3) was small (fewer than 30) for survival analysis, so we did not estimate their survival rates using our new classification. Future researchers should consider recruitment of patients with pN3 disease, although several researchers recommend against surgical interventions for $\mathrm{N} 3$ disease due to the poor survival and high comorbidity of these patients ${ }^{23}$.

In conclusion, multivariate analysis indicated that LODDS4 was a reliable prognostic indicator for patients with oral cancer. We observed stage migration in patients classified as $\mathrm{pN} 1$ or as $\mathrm{pN} 2$ with LODDS4. This study validated that prognostic utility of the newly proposed system, which incorporated LODDS with AJCC pN, compared with AJCC TNM stage. The LODDS should be considered as a future reliable lymph node measurement for $\mathrm{N}$ category in oral cancer.

\section{Material and Methods}

Data source and study population. Data were obtained from the SEER database, sponsored by the National Cancer Institute, and consists of 18 population-based cancer registries. SEER data is an open access resource from United States used for cancer-based epidemiology and survival analyses. The Surveillance Research Program, using National Cancer Institute SEER*Stat software (seer.cancer.gov/seerstat) version 8.3.2, was used to identify eligible patients. All authors provided signed authorization to access this dataset. All methods were performed in accordance with the relevant guidelines and regulations of SEER database. The study design was approved by the Ethics Committee of the Institutional Review Board of Kaohsiung Veterans General Hospital.

Patients with new diagnoses of oral cancer after major surgery, with or without adjuvant radiotherapy, were identified from 2007 to 2013.Oral cancer patients were identified using the International Classification of Disease for Oncology, third edition (ICD-O-3). The ICD-O-3 categories included in this study were cancer of the lip (C00) and oral cavity (C02-C05.0; C06). To allow comparison of results with current AJCC N categories, any oral cancer patient without a clear AJCC TNM stage was excluded. All cases were staged according to the $6^{\text {th }}$ edition AJCC system ${ }^{24}$. Patients with a previous cancer, distant metastasis at initial diagnosis, $\mathrm{pN} 2 \mathrm{c}$ disease, fewer than 10 examined LN, or who received any treatments prior to surgery (e.g. radiotherapy) were excluded. Patients with pN2c disease were also excluded because there may be confounding with the number of lymph nodes examined ${ }^{20}$. Patients with fewer than 10 lymph nodes examined may be regarded as not having received neck dissection according to our cancer center consensus. Finally, we examined the records of 3958 patients.

We examined the prognostic value of different features of neck lymph nodes in patients with oral cancer. The 3 lymph node scoring systems were:

(1) Log odds of positive lymph node (LODDS). This is calculated as $\log _{10}[(\operatorname{pnod}+0.5) /($ tnod - pnod +0.5$)]$, in which pnod is the number of positive neck lymph nodes and tnod is the total number of cervical lymph nodes examined ${ }^{25}$. In this formula, 0.5 was added to the numerator and denominator to avoid division by 0 . The cutoff points of LODDS were $35 \%$, $60 \%$, and $85 \%$ according to our previous publication ${ }^{5}$.

(2) Number of cervical lymph nodes retrieved.

This number was classified as adequate or inadequate, according to previous research ${ }^{20}$. Patients with pN0 disease with nodal yield more than 15 or those with pN1-3 disease with nodal yield more than 25 were categorized as having an adequate lymph nodes retrieval. All others were classified as having inadequate retrieval.

(3) Ratio-based lymph node system $(\mathrm{rN})$.

This ratio is calculated as the number of positive regional lymph nodes examined divided by the total number of regional lymph nodes examined. The cutoff points were 0.2 and 0.4 , as in our previous study ${ }^{21}$.

Measurements. The main endpoints were 5-year disease-specific survival (DSS) and overall survival (OS). Deaths from cancer and other conditions were extracted from the SEER database.

Other variables. Basic characteristics, including age, sex, tumor subsite, AJCC pT, cell differentiation, receipt of radiotherapy, marital status, race, and year of diagnosis were also analyzed.

Statistical analysis. All statistical analyses employed SPSS (version 15, SPSS Inc., Chicago, IL, USA). The 5 -year OS and DSS rates for different lymph node scoring systems (LODDS, lymph node yield, pN, and rN,) were compared by the Kaplan-Meier method. Survival curves were measured from the time of initial diagnosis. Death from cancer was regarded as the event in DSS analysis, and death from all causes as the event in OS analysis. In multivariate analysis, the prognostic effect of different lymph node features were analyzed after adjusting for age, sex, T stage, cell differentiation, year of diagnosis, marital status, and treatment modality. The lymph node features that remained statistically significant during multivariate analyses were selected for further analysis. We also checked whether stage migration developed among patients in different AJCC N categories and with new lymph node features. Then, we constructed a new $\mathrm{N}$ staging system by adding LODDS into the AJCC pN category to improve the accuracy of 5 year predictions of DSS and OS, and compared the new staging system with the existing AJCC staging system. Subgroups with fewer than 30 OSCC patients were not included in the analysis because the small number of patients could lead to unreliable estimates of the 5-year DSS and OS.

Three indices were used to evaluate the prediction accuracy and discriminability of each model: a linear trend chi-square test, the Akaike information criterion (AIC), and Harrell's C-statistic ${ }^{14,26,27}$. For Harrell's C-statistic, a value of 0.5 indicates a value no better than chance; a value of 0.7-0.8 indicates an acceptable model; a value of 0.8-0.9 indicates an excellent model; and a value of $0.9-1$ indicates an outstanding model. A linear trend chi-square test was used to assess monotonicity, in which a higher value indicates stronger monotonicity. Comparison of different staging system was also performed using mutltivariate analysis. A two-sided $p$-value below 0.05 was considered significant ${ }^{28}$. 


\section{References}

1. Pfister, D. G. et al. NCCN Practice Guidelines for Head and Neck Cancers. Oncology (Williston Park) 14, 163-194 (2000).

2. Rubright, W. C. et al. Risk factors for advanced-stage oral cavity cancer. Arch Otolaryngol Head Neck Surg 122, 621-626 (1996).

3. Larsen, S. R., Johansen, J., Sorensen, J. A. \& Krogdahl, A. The prognostic significance of histological features in oral squamous cell carcinoma. J Oral Pathol Med 38, 657-662, doi:10.1111/j.1600-0714.2009.00797.x (2009).

4. Ebrahimi, A. et al. Lymph node ratio as an independent prognostic factor in oral squamous cell carcinoma. Head Neck 33, 1245-1251, doi:10.1002/hed.21600 (2011).

5. Lee, C. C. et al. The Prognostic Ability of Log Odds of Positive Lymph Nodes in Oral Cavity Squamous Cell Carcinoma. Medicine (Baltimore) 94, e1069, doi:10.1097/MD.0000000000001069 (2015).

6. Lemieux, A., Kedarisetty, S., Raju, S., Orosco, R. \& Coffey, C. Lymph Node Yield as a Predictor of Survival in Pathologically Node Negative Oral Cavity Carcinoma. Otolaryngol Head Neck Surg 154, 465-472, doi:10.1177/0194599815622409 (2016).

7. Ebrahimi, A., Zhang, W. J., Gao, K. \& Clark, J. R. Nodal yield and survival in oral squamous cancer: Defining the standard of care. Cancer 117, 2917-2925, doi:10.1002/cncr.25834 (2011).

8. Divi, V. et al. Lymph Node Count From Neck Dissection Predicts Mortality in Head and Neck Cancer. J Clin Oncol. doi:10.1200/ JCO.2016.67.3863 (2016).

9. Gil, Z. et al. Lymph node density is a significant predictor of outcome in patients with oral cancer. Cancer 115, 5700-5710, doi:10.1002/cncr.24631 (2009).

10. Yang, S., Wang, D. \& Mao, C. [Prognostic significance of positive lymph node ratio in oral squamous cell carcinoma]. Zhonghua Kou Qiang Yi Xue Za Zhi 50, 696-698 (2015).

11. Sun, Z. et al. Log odds of positive lymph nodes: a novel prognostic indicator superior to the number-based and the ratio-based N category for gastric cancer patients with R0 resection. Cancer 116, 2571-2580, doi:10.1002/cncr.24989 (2010).

12. Arslan, N. C., Sokmen, S., Canda, A. E., Terzi, C. \& Sarioglu, S. The prognostic impact of the log odds of positive lymph nodes in colon cancer. Colorectal Dis 16, O386-392, doi:10.1111/codi.12702 (2014).

13. Chen, L. J., Chung, K. P., Chang, Y. J. \& Chang, Y. J. Ratio and log odds of positive lymph nodes in breast cancer patients with mastectomy. Surg Oncol 24, 239-247, doi:10.1016/j.suronc.2015.05.001 (2015).

14. Qiu, M. Z. et al. The tumor-log odds of positive lymph nodes-metastasis staging system, a promising new staging system for gastric cancer after D2 resection in China. PLoS One 7, e31736, doi:10.1371/journal.pone.0031736 (2012).

15. Lee, C. C. et al. Incorporation of log odds of positive lymph nodes into the AJCC TNM classification improves prediction of survival in oral cancer. Clin Otolaryngol. doi:10.1111/coa.12809 (2016).

16. Bernier, J. et al. Defining risk levels in locally advanced head and neck cancers: a comparative analysis of concurrent postoperative radiation plus chemotherapy trials of the EORTC (\#22931) and RTOG (\# 9501). Head Neck 27, 843-850, doi:10.1002/hed.20279 (2005).

17. Lee, C. W., Wilkinson, K. H., Sheka, A. C., Leverson, G. E. \& Kennedy, G. D. The Log Odds of Positive Lymph Nodes Stratifies and Predicts Survival of High-Risk Individuals Among Stage III Rectal Cancer Patients. Oncologist 21, 425-432, doi:10.1634/ theoncologist.2015-0441 (2016).

18. Wen, J. et al. Development and validation of a prognostic nomogram based on the log odds of positive lymph nodes (LODDS) for breast cancer. Oncotarget 7, 21046-21053, doi:10.18632/oncotarget.8091 (2016).

19. Yildiz, M. M., Petersen, I., Eigendorff, E., Schlattmann, P. \& Guntinas-Lichius, O. Which is the most suitable lymph node predictor for overall survival after primary surgery of head and neck cancer: $\mathrm{pN}$, the number or the ratio of positive lymph nodes, or log odds? J Cancer Res Clin Oncol 142, 885-893, doi:10.1007/s00432-015-2104-1 (2016).

20. Kuo, P. et al. Proposing prognostic thresholds for lymph node yield in clinically lymph node-negative and lymph node-positive cancers of the oral cavity. Cancer 122, 3624-3631, doi:10.1002/cncr.30227 (2016).

21. Chen, Y. L. et al. Prognostic influences of lymph node ratio in major cancers of Taiwan: a longitudinal study from a single cancer center. Journal of cancer research and clinical oncology 141, 333-343, doi:10.1007/s00432-014-1810-4 (2015).

22. Angrist JD IG, R. D. Indentification of causal effects using instrumental variables. J Am Stat Assoc 444-455 (1996).

23. Karakaya, E. et al. Outcomes following chemoradiotherapy for N3 head and neck squamous cell carcinoma without a planned neck dissection. Oral oncology 49, 55-59, doi:10.1016/j.oraloncology.2012.07.010 (2013).

24. Edge, S. B. \& Compton, C. C. The American Joint Committee on Cancer: the 7th edition of the AJCC cancer staging manual and the future of TNM. Ann Surg Oncol 17, 1471-1474, doi:10.1245/s10434-010-0985-4 (2010).

25. Wang, J., Hassett, J. M., Dayton, M. T. \& Kulaylat, M. N. The prognostic superiority of log odds of positive lymph nodes in stage III colon cancer. J Gastrointest Surg 12, 1790-1796, doi:10.1007/s11605-008-0651-3 (2008).

26. Wang, W. et al. Tumor-ratio-metastasis staging system as an alternative to the 7th edition UICC TNM system in gastric cancer after D2 resection-results of a single-institution study of 1343 Chinese patients. Ann Oncol 22, 2049-2056, doi:10.1093/annonc/mdq716 (2011).

27. DeLong, E. R., DeLong, D. M. \& Clarke-Pearson, D. L. Comparing the areas under two or more correlated receiver operating characteristic curves: a nonparametric approach. Biometrics 44, 837-845 (1988).

28. McGinn, T. G. et al. Users' guides to the medical literature: XXII: how to use articles about clinical decision rules. Evidence-Based Medicine Working Group. JAMA 284, 79-84, doi:jml90005 [pii] (2000).

\section{Acknowledgements}

The authors acknowledge the efforts of the Surveillance, Epidemiology, and End Results (SEER) Program tumor registries in the creation of the SEER database. The interpretation of these data is the sole responsibility of the authors. This study was partly supported by grant DTCRD103(2)-I-20.

\section{Author Contributions}

C.C.L., Y.C.S., S.K.H., P.C.C., C.I.H., W.L.H., Y.W.L. and C.C.Y. designed the research; C.C.L., Y.C.S. and C.C.Y. performed the research; C.C.L. and C.C.Y. analyzed data and wrote the manuscript. All authors reviewed the manuscript.

\section{Additional Information \\ Supplementary information accompanies this paper at doi:10.1038/s41598-017-06452-0}

Competing Interests: The authors declare that they have no competing interests.

Publisher's note: Springer Nature remains neutral with regard to jurisdictional claims in published maps and institutional affiliations. 
(i) Open Access This article is licensed under a Creative Commons Attribution 4.0 International License, which permits use, sharing, adaptation, distribution and reproduction in any medium or format, as long as you give appropriate credit to the original author(s) and the source, provide a link to the Creative Commons license, and indicate if changes were made. The images or other third party material in this article are included in the article's Creative Commons license, unless indicated otherwise in a credit line to the material. If material is not included in the article's Creative Commons license and your intended use is not permitted by statutory regulation or exceeds the permitted use, you will need to obtain permission directly from the copyright holder. To view a copy of this license, visit http://creativecommons.org/licenses/by/4.0/.

(C) The Author(s) 2017 